\title{
Teledermatology in São Paolo, Brazil
}

\author{
Mara Giavina-Bianchi, $\mathrm{PhD}$ \\ Telemedicine Department, Hospital Israelita Albert Einstein, São Paulo, Brazil
}

\section{Corresponding Author:}

Mara Giavina-Bianchi, PhD

Telemedicine Department

Hospital Israelita Albert Einstein

Av Albert Einstein

627 - Jardim Leonor

São Paulo, 05652-900

Brazil

Phone: 551121511233

Email: marahgbianchi@gmail.com

\section{Abstract}

Background: There are places in the world where access to dermatologists can be very challenging and general practitioners may not be well trained in the diagnosis and treatment of skin conditions. Store-and-forward teledermatology may improve access to specialty care, provide accurate diagnoses, and reduce time to treatment, resulting in high patient satisfaction. The early detection and timely treatment of severe skin diseases could prevent adverse health outcomes and death. On the other hand, some skin conditions such as mild atopic dermatitis, acne, and fungal infections could be managed within primary care using teledermatology.

Objective: We aimed to (1) evaluate the proportion of individuals who could be assessed in primary care using teledermatology and how this affects the waiting time for an in-person dermatologist appointment and (2) assess the most frequent dermatoses according to demographic data and referrals made by the teledermatologist.

Methods: A cross-sectional retrospective study, involving 30,976 individuals and 55,624 skin lesions, was conducted from July 2017-July 2018 in the city of São Paulo. We assessed the frequency of diagnoses and referrals to biopsy, in-person dermatologists, or primary care, and compared the waiting time for an in-person dermatologist appointment before and after the teledermatology implementation.

Results: We found that $53 \%$ of the patients were managed by the primary care physician, $43 \%$ were referred to in-person dermatologists, and $4 \%$ were referred directly to biopsy, leading to a reduction in waiting time for in-person appointments of $78 \%$ when compared to the previous period (from 6.7 months to 1.5 months). The most frequent diseases were melanocytic nevus, seborrheic keratosis, acne, benign neoplasms, onychomycosis, atopic dermatitis, solar lentigo, melasma, xerosis, and epidermoid cyst, with significant differences according to sex, age, and referrals (Multimedia Appendix 1A,B).

Conclusions: The use of teledermatology as a triage tool significantly reduced the waiting time for in-person visits, improving health care access and using public resources wisely. Knowledge of sex, age, diagnoses, and treatment of common skin conditions can enable the creation of public policies for prevention and orientation of the population, as it can be used to train general physicians to address such cases.

Conflicts of Interest: None declared.

(iproc 2022;8(1):e36899) doi: 10.2196/36899

\section{KEYWORDS}

teledermatology; common skin lesions; primary care attention

\section{Multimedia Appendix 1}

(A) Most frequent skin diseases diagnosed and (B) triage results by disease type.

[PNG File, 146 KB-Multimedia Appendix 1] 
Edited by T Derrick; this is a non-peer-reviewed article. Submitted 28.01.22; accepted 28.01.22; published 10.02.22.

Please cite as:

Giavina-Bianchi $M$

Teledermatology in São Paolo, Brazil

iproc 2022;8(1):e36899

URL: https://www.iproc.org/2022/1/e36899

doi: $\underline{10.2196 / 36899}$

PMID:

CMara Giavina-Bianchi. Originally published in Iproceedings (https://www.iproc.org), 10.02.2022. This is an open-access article distributed under the terms of the Creative Commons Attribution License (https://creativecommons.org/licenses/by/4.0/), which permits unrestricted use, distribution, and reproduction in any medium, provided the original work, first published in Iproceedings, is properly cited. The complete bibliographic information, a link to the original publication on https://www.iproc.org/, as well as this copyright and license information must be included. 